\title{
EQUIPMENT PROTECTION ISSUES FOR FAST EXTRACTION FROM SPS TO LHC
}

\author{
B.Goddard, E.Carlier, P.Knaus and J.Uythoven, CERN, Geneva, Switzerland
}

\begin{abstract}
The proton and lead ion beams for the Large Hadron Collider (LHC) will be extracted from the Super Proton Synchrotron (SPS) in the Long Straight Sections LSS6 and LSS4, and transferred to the LHC rings 1 and 2 respectively via the transfer lines TI 2 and TI 8 . Single turn 'fast' extraction will be used in both cases. In LSS4 a new extraction system will be installed using the present design of electromagnetic septum and extraction kickers. In LSS6 the extraction system will be adapted for fast extraction using the existing septa and modified kickers. The high brightness LHC beam presents problems of protection for both existing and new equipment. Solutions are proposed and implications discussed.
\end{abstract}

\section{INTRODUCTION}

In the LSS6 extraction channel of the SPS, in addition to the fast extraction (FE) to fill LHC ring 1, resonant (slow) extraction (SE) will continue to service CERN's West Experimental Area. SE uses a thin electrostatic septum (ZS), followed by magnetic septa (MST and MSE) to move the beam out of the aperture. For LHC FE, an extraction kicker magnet (MKE) deflects the whole beam across the first septum. The LSS6 FE is shown in figure. 1 (all trajectories are given as a $\pm 3 \sigma$ envelope).

In LSS4, FE is the only operating mode [1]. A kicker MKE deflects the beam directly across the magnetic septum MSE. The LSS4 FE is shown in figure 2.

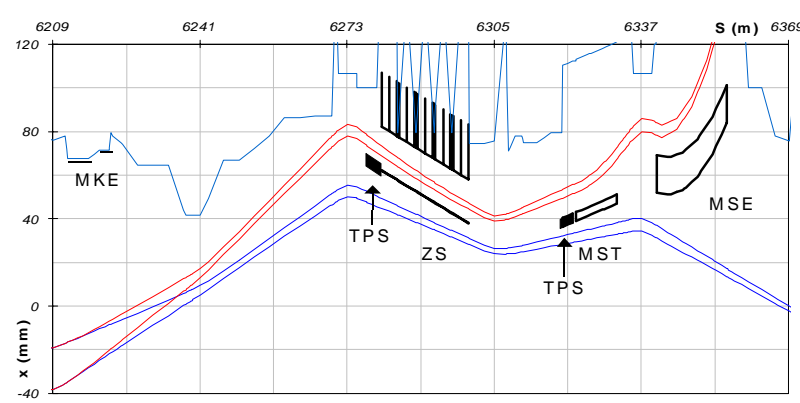

Figure 1.FE in LSS6 (uses MKE, ZS, MST and MSE).

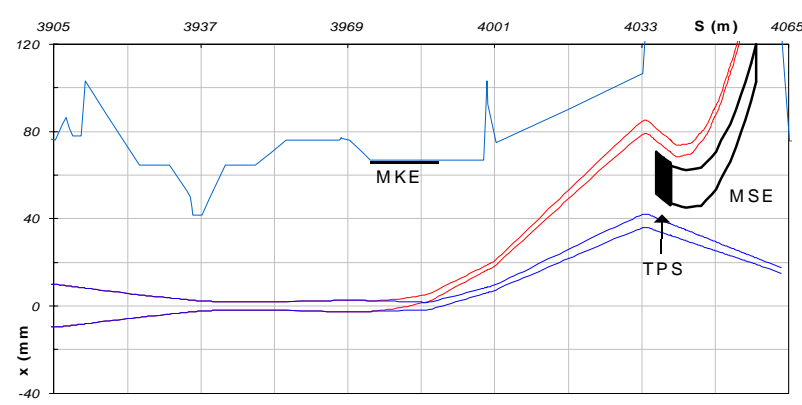

Figure 2. FE in LSS4 (uses only MKE and MSE).
The relevant parameters of the LHC proton beam at extraction in the SPS are given in table 1 for a bunch train comprising 4 injected batches (values for LHC 'ultimate' intensities are given in parentheses).

Table 1: Beam parameters at extraction (4 PS batches)

\begin{tabular}{|l|l|}
\hline Parameter & Value \\
\hline Momentum $p$ & $450 \mathrm{GeV} / \mathrm{c}$ \\
\hline Normalised emittance $\varepsilon_{N}$ & $3.5 \mu \mathrm{rad}$ \\
\hline Total number of bunches & 288 \\
\hline Overall length of bunch train & $7.8 \mu \mathrm{s}$ \\
\hline Particles per bunch & $1.110^{11}\left(1.710^{11}\right)$ \\
\hline Total particles per bunch train & $3.210^{13}\left(4.910^{13}\right)$ \\
\hline Stored beam energy & $2.7 \mathrm{MJ} \quad(3.5 \mathrm{MJ})$ \\
\hline
\end{tabular}

\section{FAILURE MODES}

For a well-set up and correctly operating (and operated) machine, FE has essentially no beam losses. However, several independent failure modes do exist which could lead to impact of the circulating or extracted beam on septum elements. These include errors in: position of circulating beam; bumper magnet fields; kicker fields or timing; septum fields; septum alignment. In addition, during the setting-up of the extraction, there may be continued losses on the septum elements as part of the optimisation process. For the purposes of this analysis, the worst-case scenario for the protection requirements is taken as a full (4 PS batches) LHC beam at $450 \mathrm{GeV} / \mathrm{c}$ striking the centre of the element under consideration.

\section{SIMULATION RESULTS}

Simulations of beam impact on various combinations of septum elements and protection devices (TPS) were made using FLUKA [2]. The lengths and material compositions of the TPS were varied to optimise the protection.

\subsection{LSS4 extraction}

In LSS4 $3.25 \mathrm{~m}$ is available for a protection element (TPS), limited by the geometrical requirements imposed by the SPS lattice [1]. Various constructions were simulated to optimise the protection level - the figure of merit being the temperature reached in the downstream copper septum coil which ideally should remain below $100^{\circ} \mathrm{C}$. Optimum protection was afforded by a $2.35 \mathrm{~m}$ length of high density graphite, followed by $0.9 \mathrm{~m}$ of aluminium. Figure. 3 shows the simulated effect of an ultimate LHC beam impact on such an element. The graphite reaches $704^{\circ} \mathrm{C}$, the aluminium reaches $196^{\circ} \mathrm{C}$ and the MSE coil temperature reaches $77^{\circ} \mathrm{C}$. For the LHC 
nominal beam intensity, peak temperatures in the $\mathrm{C} / \mathrm{Al} / \mathrm{Cu}$ are 463,136 and $59^{\circ} \mathrm{C}$, respectively.

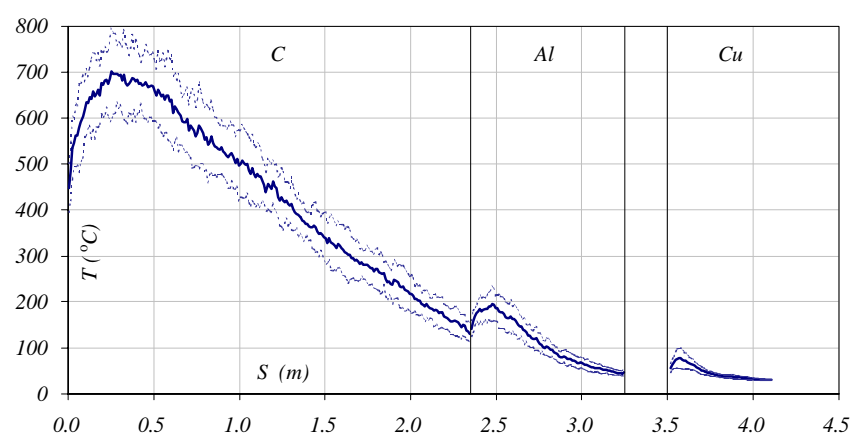

Figure 3. Temperature of C/Al TPS and first part of $\mathrm{Cu}$ septum coil, for LHC ultimate beam impact $( \pm 1 \sigma$ limits are shown for 10 simulation runs of 1000 protons).

\subsection{LSS6 extraction}

In LSS6 the protection of the extraction elements is problematic. Protection of the magnetic septa will be accomplished by an upgrade of the existing TPSS device element installed upstream of the MST. However, in the reference extraction scheme, the ZS is the first septum element in the LHC extraction. Simulations of the impact of the LHC beam on the ZS wires were made [3], which showed that the wires would be destroyed for a beam intensity of only $210^{13}$. This was also confirmed experimentally in the SPS [4]. The feasibility of protecting the ZS wires with a mobile $5 \mathrm{~mm}$ wide TPS was then studied. Simulations showed that, although the wire temperature can easily be reduced to acceptable levels of a few hundred degrees, a major difficulty is the lack of containment offered by such a thin object. Figure 4 shows the density of the charged particles incident on the high voltage cathode of the first ZS, which will trigger a high voltage discharge and also risk causing permanent damage to the fragile surface of the high voltage cathodes (the instantaneous temperature rise is about $30^{\circ} \mathrm{C}$ ).

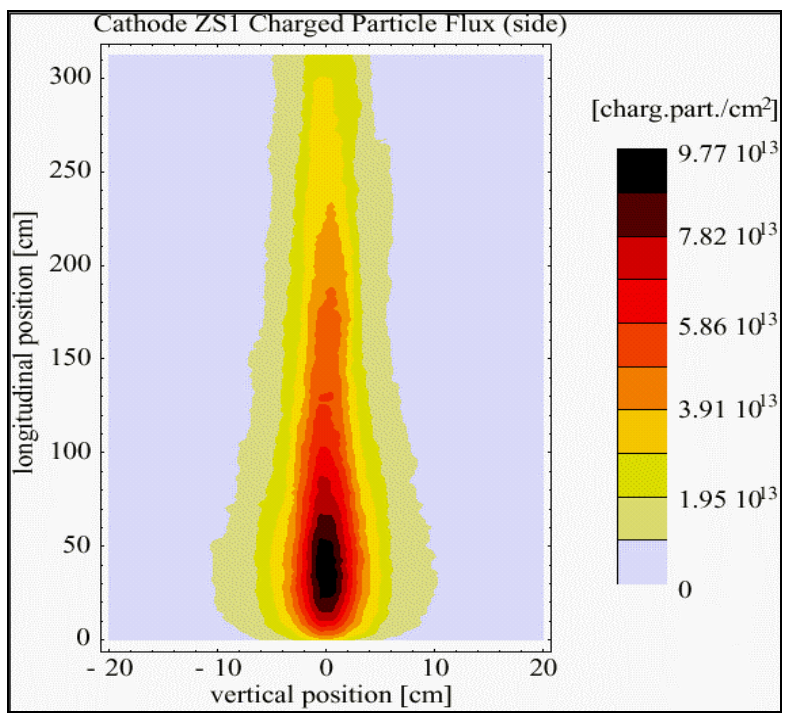

Figure 4. Charged particle density on ZS1 cathode after impact of LHC ultimate beam on TPS.
Another drawback is the need to move the protection element into the beam before each LHC injection and to remove it for resonant extraction. Finally, the only currently acceptable engineering material for such an object is graphite, the use of which adjacent to such high voltage elements poses particular risks of contamination and serious performance deterioration for the $\mathrm{ZS}$.

\section{ALTERNATIVE EXTRACTION IN LSS6}

Alternatives were explored with a view to improving the reliability and the safety of the LSS6 LHC extraction. The most promising is to use the space upstream of the first ZS foreseen for the TPS, for two extraction kicker magnets, figure 5 . The $\mathrm{ZS}$ is then dispensed with entirely for the LHC extraction, see figure 6. There are many advantages to such a scheme, which in effect decouples the critical MKE and ZS elements used for fast and slow extraction respectively, and also removes the need for a mobile TPS upstream of the ZS. The main disadvantage is that a new, low-impedance, variant of MKE magnet and pulse forming network need to be developed and built, with larger horizontal aperture and a bigger deflection. Such a magnet seems feasible and a detailed design is now being studied.

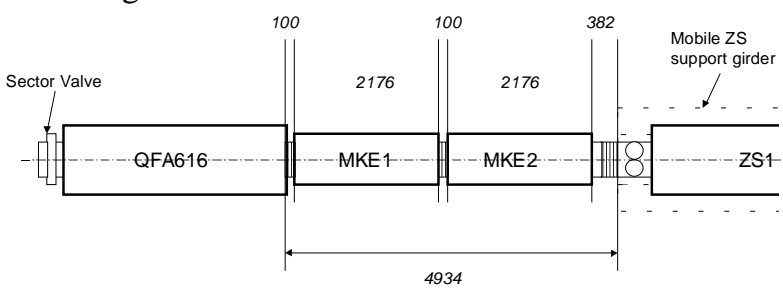

Figure 5. Schematic plan view showing two MKE magnets upstream of ZS1 in LSS6 (dimensions in $\mathrm{mm}$ ).

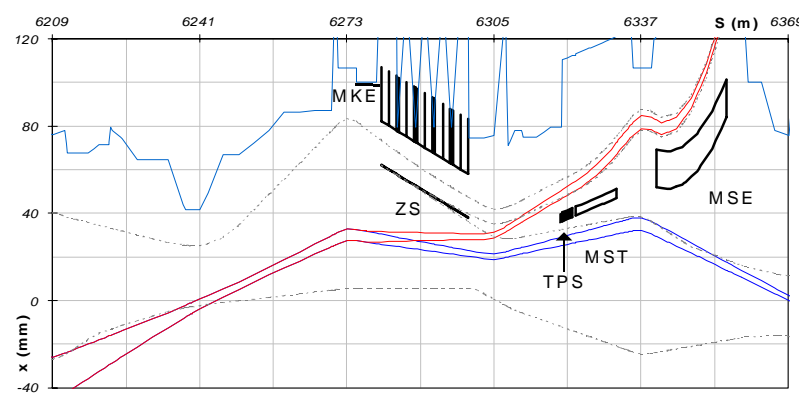

Figure 6. Alternative FE for LHC in LSS6, using only MST and MSE septa. The envelope of the resonant extraction is also indicated (dashed) for illustration.

\section{EFFECTS ON SPS SE OPERATION}

For SE operation, the reference scheme for LSS6 and the alternative, described above, differ only in the need to remove the mobile TPS before extraction for the former. Both versions require changes to the layout by suppressing the first $\mathrm{ZS}$ and the addition of a $\mathrm{ZS}$ in position 6. The most important expected effects of this change, already implemented for the 2001 run, are on the aperture, the effective strength of the ZS and the extraction settings. 


\subsection{Aperture}

The changes will modify the horizontal and vertical apertures, as a result of the opening in the ZS itself and the new position of the TCE collimator element. The minimum vertical aperture of the extraction channel decreases slightly from $5.8 \sigma$ to $5.4 \sigma$. However, the new figure is still well above the SPS machine limit of $4.9 \sigma$, determined by the beam dump TIDVG, and remains comparable to the $5.6 \sigma$ aperture of the main machine dipoles.

\subsection{ZS strength}

The downstream displacement of the ZS septum reduces the opening produced at the MST septum to $10.8 \mathrm{~mm}$, which from previous experience is acceptable. One important drawback is, however, the reduction in the operational margin in the event of an equipment fault.

\subsection{Shared slow extraction}

With the new layout the separatrices of the beam in resonance no longer coincide at the entrances of the ZS in LSS2 and LSS6 for the shared slow extraction mode [5]. Simulations with the new layout show that the effect on the extraction setting-up and operation will be small in terms of beam losses and extraction setting up and adjustment.

\section{INTERLOCKS AND VETOES}

Although the physical protection devices will be installed to guard against worst-case failure, the main protection for the extraction equipment (and indeed that of the SPS machine and the transfer lines) will come from a comprehensive interlock/veto system. This machine protection system will, in the multi-cycling operational environment of the SPS, need to make use of critical information on equipment state, beam parameters, beam requests and operational constraints to dynamically enable, disable and abort safely the SPS beams.

The system must take into account the status of the items listed above, together with other information concerning the SPS, transfer line and Client systems (CNGS, LHC, experimental areas). Only if all conditions are correct can the extraction proceed. With this concept the interlock matrix is conditioned actively by the SPS status and by other inputs, as compatibility with machine multi-cycling is a prerequisite. This means that the response to different fault conditions will vary according to the machine state - for example an internal fault on the LSS6 MKE kicker system must veto the fast extraction in this zone, but should allow extraction via LSS4 and also resonant extraction in LSS6.

This extraction interlock/veto system, which remains to be designed, must be integrated into the new SPS interlock system and future SPS control system. Basic inputs to such a system and some possible actions are shown schematically in figure 7 .

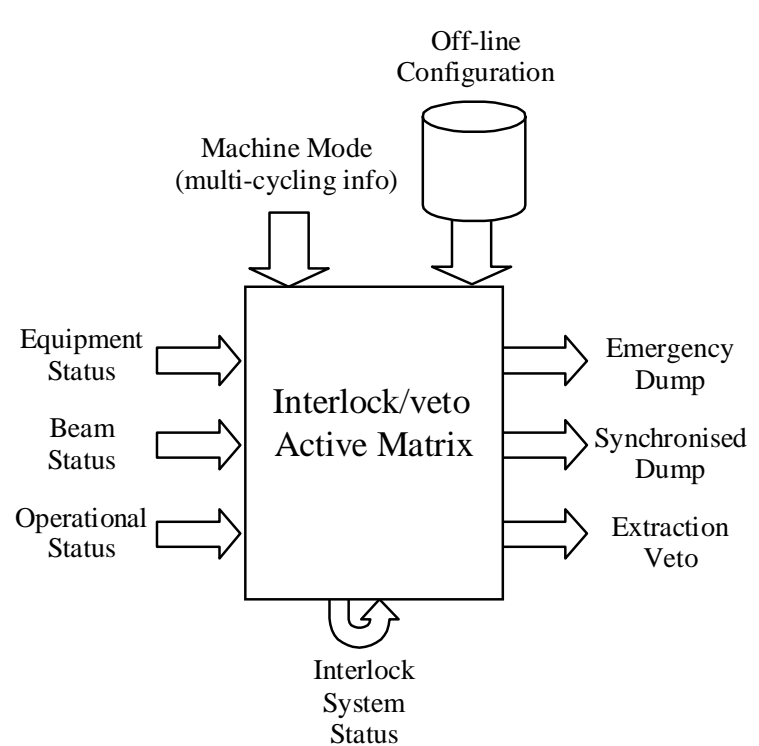

Figure 7. Synoptic for 'active matrix' based extraction interlock/veto system.

\section{CONCLUSIONS}

The extraction systems in the SPS require protection from the high brightness fast extracted LHC beams. The protection of the magnetic septa in LSS4 and LSS6 will be accomplished using a composite element to dilute the energy deposited to acceptable levels. A similar protection of the ZS septum in LSS6 presents many problems; the preferred solution is now to modify the layout and hardware to allow fast extraction without using the ZS. In addition to these system-specific changes, a comprehensive SPS interlock/veto system for equipment protection and beam quality control is necessary [6] and still awaiting development.

\section{ACKNOWLEDGEMENTS}

The substantial contributions of several CERN colleagues to this work are gratefully acknowledged, in particular B.Balhan, E.Gaxiola, M.Gyr, J.Ramillon and E.Weisse.

\section{REFERENCES}

[1] P.Knaus et al., "The new SPS extraction channel for LHC and CNGS", EPAC '00, Vienna, 2000.

[2] A. Fasso, A. Ferrari, J. Ranft, P. Sala, "Fluka 99 User's Manual", 1999.

[3] B.Goddard and P.Knaus, "Beam loss damage in a wire septum", EPAC '00, Vienna, 2000.

[4] P.Knaus et al., CERN SL-MD Note, to be published.

[5] M.Gyr et al., "Simultaneous slow resonant extractions from the SPS with horizontal tune-split," IEEE Trans. Nucl. Sci. 32, pp.2997-2999, 1985.

[6] P.Collier, "Controlling the beam for extraction", CERN Workshop on LHC, Chamonix, 2001. 